\title{
Staying Calm and Well in the Midst of the COVID-19 Storm: One University's Longitudinal Outcomes Evaluation of a Mental Health and Well-Being Webinar Series
}

\author{
Bernadette Mazurek Melnyk, PhD, APRN-CNP, FAANP, FNAP, FAAN \\ The Ohio State University \\ Megan Amaya, PhD, CHES \\ The Ohio State University \\ Andreanna Pavan Hsieh, MPH \\ The Ohio State University \\ Rebecca Momany, MA \\ The Ohio State University \\ Josh Winn, MS \\ The Ohio State University \\ Logan J. Forbes \\ The Ohio State University
}

\begin{abstract}
Background: As the COVID-19 pandemic began, wellness leadership at a large Mid-west public university anticipated an increase in mental health problems among faculty and staff. A two-part weekly webinar series entitled "Staying Calm and Well in the Midst of the COVID-19 Storm" was launched to assist faculty and staff in coping with stressful issues related to the pandemic and enhancing their well-being. This series provided the university community with evidence-based knowledge, skills, and resources to enhance their ability in taking good self-care.

Aim: To assess participant mental health outcomes during the two-part mental health and well-being educational webinar series over time.

Methods: A longitudinal outcomes evaluation was conducted. Evaluation data was obtained from the participants via an anonymous post-webinar survey, which included questions from the Patient-Health Questionnaire-4 (PHQ-4) to assess symptoms of anxiety and depression. The first series was conducted over the course of 8 weeks and the second series took place over 7 weeks. Descriptive statistics were used to assess responses to the PHQ-4 over time to evaluate outcomes of the webinar series. An open-ended question also asked participants about the 2 to 3 biggest concerns and worries they had at that moment.

Results: The average amount of survey responses received were 1,470.25 (SD $= \pm 240.3)$ for Series One and 926.4 (SD $= \pm 164.3$ ) for Series Two. Ninety-five percent of participants put the skills they
\end{abstract}


learned during both series into practice. When participants reported experiencing anxiety or depressive symptoms, they most frequently reported experiencing them several days a week. The number of participants reporting no depressive or anxiety symptoms increased as the webinar series progressed over time. The types of concerns and worries reported varied across time.

Conclusion: The Staying Calm and Well During the COVID-19 Pandemic webinar participants had a reduction in symptoms associated with anxiety and depression after completing the series.

Submitted 25 May 2021: accepted 28 September 2021

Keywords: COVID-19, mental health, faculty, university or universities, outcomes evaluation

The COVID-19 public health crisis adversely effects the health, safety, and well-being of both individuals and communities through such drivers as stigma, emotional isolation, school closures, financial strain, and inadequate access to needed resources (Pfefferbaum \& North, 2020). These adverse effects may translate into a range of emotional responses (e.g., depression and anxiety), unhealthy behaviors (e.g., reduced physical activity, emotional eating, and excessive alcohol and substance use), and noncompliance with public health directives. An April 2020 poll conducted by the Kaiser Family Foundation (KFF) found that 7 in 10 Americans (72\%) reported their lives as being disrupted "a lot" or "some" by the coronavirus outbreak (Kirzinger, Kearney, Hamel, \& Brodie, 2020). Nearly half $(45 \%)$ of those polled in the United States reported that their mental health had been negatively impacted due to worry and stress over the virus. The full aftermath of the mental and physical health problems due to the virus have yet to become fully realized, but a Morbidity and Mortality Weekly Report (Czeisler et al., 2020) stated that, in relation to COVID-19, the prevalence of anxiety disorders in adults was 3x higher and the prevalence of depression was 4x higher than values reported in the second quarter of 2019. In a recent systematic review of 66 studies, findings indicated decreases in physical activity and increased in sedentary behavior across multiple populations (adults, children, and those with a medical condition) in conjunction with COVID-19 restrictions (Stockwell et al., 2021). Other studies have found an increase in unhealthy eating as people attempt to cope with the pandemic (Bin Zarah, Enriquez-Marulanda, \& Andrade, 2020; Di Renzo et al., 2020; Marty, De Lauzon-Guillain, Labesse, Nicklaus, 2021).

The reported increases in mental health disorders and reductions in physical activity are alarming. Mental health and level of physical activity are innately tied together, and both impact the likelihood of succumbing to a chronic disease later in life. Individuals with untreated or unmitigated depression and anxiety have an increased risk of cardiovascular disease, as do those who are sedentary (Centers for Disease Control and Prevention, 2020). Consequently, those impacted have substantial decreases in quality of life, and up to half of all premature deaths are related to modifiable behavioral factors such as unhealthy eating, inactivity, and tobacco use (Institute of Medicine \& National Research Council, 2015).

While chronic diseases can be prevented or have their symptoms alleviated through healthy lifestyle behaviors (Barbaresko, Rienks, \& Nöthlings, 2018; Uusitupa et al., 2019), making sustained behavior change is challenging. According to the Social Ecological Model (SEM), for healthy behavior change to occur, an individual must be educated on how to make the change and be sufficiently motivated (Sallis, Ownen, \& Fisher, 2015). Furthermore, an individual's environment and culture (ecology) must provide the appropriate policies and support to enhance one's behavior change ability. Unfortunately, most people do not make needed lifestyle changes unless a crisis happens or 
their emotions are raised (Melnyk \& Neale, 2019). To meet both components of the SEM, hospital systems and institutions of higher education have been hiring Chief Wellness Officers (CWO) to spearhead population improvements in health and well-being by building wellness cultures, implementing wellness programming, and tracking health and well-being outcomes of their employees (Kishore et al., 2018; Ripp \& Shanafelt, 2020). Worksite wellness programming can assist in promoting and educating about good self-care, thus improving employee health outcomes and productivity (Kent, Goetzel, Roemer, Prasad, \& Freundlich, 2016).

When COVID-19 shutdowns and restrictions began in the United States, the Chief Wellness Officer at a large public land grant university in the Mid-west and her university-wide wellness team wanted to ensure that faculty and staff received needed support for having their COVID-19 concerns addressed. In March of 2020, it became clear to university wellness leadership that additional mental health resources would be needed for supporting the university community. Therefore, the CWO and her team established the ongoing Staying Calm and Well in the Midst of the COVID19 Storm webinar series to provide the university community with an evidence-based educational and skills building webinar series along with resources for self-care. The weekly webinars had a post-webinar survey that included validated scales to assess participant mental health and an open-ended question that asked about worries and concerns. By tracking and assessing both aspects, the wellness team could determine if the programing was improving mental health. Themes from the open-ended question about worries and concerns were used to guide future webinar series content.

\section{METHODS}

The purpose of this longitudinal based outcomes evaluation was to assess participant mental health outcomes in a two-part mental health and well-being educational and skills building webinar series over time. Evaluation data was obtained from the participants via a post-webinar survey. The first series took place over the course of 8 weeks and the second series took place over 7 weeks. The webinar series is ongoing; however, this manuscript reviews the findings from the first two webinar series. Series One took place April 2020 - May 2020 and Series Two took place August 2020 - September 2020. The Institutional Review Board determined that this was a quality improvement project with anonymous evaluation data from participants, therefore, it was determined exempt from research review.

\section{Participants}

Participants were invited to attend the series through emails and postings in the university's daily newsletter. The study sample included all university faculty and staff (regardless of full-time equivalent [FTE]); graduate, professional and undergraduate students; and other members of the community (non-Ohio State participants). Attendees selfselected to attend and listen to the educational webinars.

\section{Participation Incentives}

As an incentive for participation, Your Plan for Health (YP4H) points were offered to all faculty and staff attendees. $\mathrm{YP} 4 \mathrm{H}$ is a complimentary, award winning on-line wellness portal with programs and resources available to university 
faculty and staff. Non-Ohio State participants were not eligible for YP4H points. YP4H points accumulate, and once enough are obtained they are added as premium credit to the participants Health Reimbursement Account.

\section{Webinar Details}

Any faculty, staff, and students at the university as well as members of the community were able to listen in and attend the webinars. Webinars took place over the course of 7-8 weeks. Live webinars occurred every Wednesday at noon and lasted for 30 minutes. A recorded version of each webinar was made available on the Staying Well webpage so that those who were not able to attend "live" could access the information at a more suitable time. The recorded webinar and links to the post-webinar questions were entered on the Staying Well webpage within a few hours following each live webinar. Participants were also emailed a link to the posted recordings and questions the same afternoon. The survey questions remained open for a week per webinar.

The purpose of the webinar series was to equip participants with important knowledge and skills to sustain their mental health and overall well-being during the COVID-19 pandemic. Table 1 displays the topics covered in series one and series two. Each session was delivered by expert faculty. A skills building activity was included at the end of each webinar to encourage participants to put what they learned into practice. Practical evidence-based tactics and resources were provided in each session. The skills learned were designed to build participant strengths to last well beyond the pandemic.

Table 1. Staying Calm and Well Webinar Series' Topic

\begin{tabular}{|l|}
\hline Series One Weekly Topics \\
\hline Stress Busters: Using Cognitive-Behavioral Skills to Allay Anxiety \& Depression \\
\hline Staying Physically Active While Home: Tips and Tricks \\
\hline Be Here Now: Mindfulness Works! \\
\hline Sleep Soundly During the COVID Pandemic: You Can Do It! \\
\hline Physically Distancing and Loneliness: How to Stay Socially Connected \\
\hline Building Resiliency Skills: A Necessity in the COVID-19 Epidemic \\
\hline Eating Healthy and Balanced in Stressful Times: Why it Matters \\
\hline Gratitude and Positivity: A Dynamic Duo in the Midst of the COVID-19 Storm \\
\hline Series Two Weekly Topics \\
\hline Positive Psychology: Flourishing during "Character Building" Times \\
\hline Coping with Change in the Workplace \\
\hline Financial Well-Being: Managing Credit and Debt Wisely \\
\hline Boosting Immunity \& Sustaining Energy with Healthy Lifestyle Behaviors: Tips \& Tricks \\
\hline Helpful Strategies for Dealing with Grief \\
\hline Healthy Nutrition Practices: Why Now \& How? \\
\hline Preventing, Recognizing \& Dealing with Anxiety \& Depression in Children \& Teens: A Primer for Parents \\
\hline
\end{tabular}

Note. These lectures are available for free access at https://u.osu.edu/keepcalmcovid19/recorded-webinars/ and https://u.osu.edu/keepcalmcovid192/recorded-webinars/ 


\section{Weekly Post-Webinar Survey Measures}

Completion of the post-webinar survey was voluntary, anonymous, and sent via a secure, online survey tool (Qualtrics, Prove, UT) for collection of data each week. Attendees were emailed a link to the post-webinar survey a few hours after the live webinar. Attendees were asked to complete the post-webinar survey within seven days. Ultimately, it was the participant's decision to complete the post-webinar survey or not. The same survey measures were used for all weeks of the program, i.e., every week following the webinar on Wednesday.

Patient Health Questionnaire-4 (PHQ-4). The PHQ-4 (Löwe et al., 2010) is a 4-item valid and reliable scale that combines the two-item Generalized Anxiety Disorder-2 (GAD-2; Kroenke, Spitzer, Williams, Monahan, \& Löwe, 2007) and the Patient Health Questionnaire-2 (PHQ-2; Kroenke, Spitzer, \& Williams, 2003) to rapidly screen for symptoms of anxiety and depression experienced over the past two weeks. The anxiety items inquire about "feeling nervous, anxious or on edge" and "not being able to stop or control worry." The depression items inquire about being bothered by "little interest or pleasure in doing things" and "feeling down, depressed, or hopeless." The responses to the items are "not all," "several days a week," "more than half the days," and "nearly every day." The published Cronbach's $\alpha$ for the PHQ-4 is 0.82 for the general population (Löwe et al., 2010).

Open Ended Worry Question. Participants were asked to provide a response to the open-ended question "What are the 2 to 3 biggest concerns/worries that you have right now?" This question was used to assess changes in worry themes over the course of the pandemic and to inform the CWO as to what future webinar topics would be helpful to the participants.

\section{Data Analysis}

Descriptive statistics (i.e., frequencies and percentages) were used to assess demographics (i.e., association with the University) and responses to the PHQ-2 and GAD-2 at each week to examine data distribution. To identify worry patterns across the dataset, a cursory themed analysis was conducted for the open-ended worry question. Responses were reviewed by two study team members for common recurring themes. Microsoft Excel was used to analyze and compare data.

\section{RESULTS}

The amount of survey responses received varied by week as did the demographics (Table 2), however, the average amount of responses received were 1,470.25 (SD = \pm 240.3) for Series One and $926.4(S D= \pm$ 164.3) for Series Two. In both series, most participants were either a university faculty or staff member (Series One $M=1158$ [87.5\%], SD $= \pm 152$; Series Two $M=867$ [85.6\%], SD = 152) or the spouse of a university faculty or staff member (Series One $M=44(47.4 \%), S D= \pm 7$; Series Two $M=27$ (75.6\%), $S D= \pm 7)$. In Series One, 95.8\% $(n=1,166)$ of participants put the skills they learned into practice. This rate was almost identical for Series Two, with 95.7\% $(n=657)$ of participants indicating they put the skills they learned into practice. 
Table 2. Participant Association with the University by Week

\begin{tabular}{|c|c|c|c|c|c|c|c|c|c|c|c|c|c|c|c|c|}
\hline & \multicolumn{2}{|c|}{ Week 1} & \multicolumn{2}{|c|}{ Week 2} & \multicolumn{2}{|c|}{ Week 3} & \multicolumn{2}{|c|}{ Week 4} & \multicolumn{2}{|c|}{ Week 5} & \multicolumn{2}{|c|}{ Week 6} & \multicolumn{2}{|c|}{ Week 7} & \multicolumn{2}{|c|}{ Week 8} \\
\hline & $\mathrm{n}$ & $\%$ & $\mathrm{n}$ & $\%$ & $\mathrm{n}$ & $\%$ & $\mathrm{n}$ & $\%$ & $\mathrm{n}$ & $\%$ & $\mathrm{n}$ & $\%$ & $\mathrm{n}$ & $\%$ & $\mathrm{n}$ & $\%$ \\
\hline $\begin{array}{l}\text { Series } \\
\text { One } \\
\end{array}$ & & & & & & & & & & & & & & & & \\
\hline $\begin{array}{l}\text { Employed } \\
\text { by Ohio } \\
\text { State }\end{array}$ & 1180 & 85.2 & 1487 & 89.4 & 1196 & 89.0 & 1164 & 87.3 & 1130 & 86.3 & 984 & 87.3 & 1035 & 87.7 & 1090 & 87.9 \\
\hline $\begin{array}{l}\text { An Ohio } \\
\text { State } \\
\text { Student }\end{array}$ & 46 & 3.3 & 52 & 3.1 & 41 & 3.1 & 40 & 3.0 & 43 & 3.3 & 28 & 2.5 & 29 & 2.5 & 35 & 2.8 \\
\hline $\begin{array}{l}\text { Not } \\
\text { Affiliated } \\
\text { with Ohio } \\
\text { State }\end{array}$ & 159 & 11.5 & 124 & 7.5 & 108 & 8.0 & 92 & 6.9 & 88 & 6.7 & 74 & 6.8 & 78 & 6.6 & 77 & 6.2 \\
\hline $\begin{array}{l}\text { A Spouse } \\
\text { of an Ohio } \\
\text { State } \\
\text { Employee }\end{array}$ & 32 & 27.8 & 55 & 44.4 & 48 & 44.9 & 45 & 48.9 & 44 & 50 & 38 & 51.3 & 44 & 56.4 & 43 & 55.8 \\
\hline $\begin{array}{l}\text { Series } \\
\text { Two }\end{array}$ & & & & & & & & & & & & & & & & \\
\hline $\begin{array}{l}\text { Employed } \\
\text { by Ohio } \\
\text { State } \\
\end{array}$ & 1073 & 85.3 & 986 & 85.3 & 905 & 85.2 & 914 & 86.0 & 835 & 86.3 & 737 & 85.3 & 621 & 86.0 & $\mathrm{X}$ & $\mathrm{X}$ \\
\hline $\begin{array}{l}\text { An Ohio } \\
\text { State } \\
\text { Student }\end{array}$ & 49 & 3.9 & 49 & 4.2 & 41 & 3.86 & 40 & 3.8 & 39 & 4.0 & 33 & 3.8 & 26 & 3.6 & $\mathrm{X}$ & $\mathrm{X}$ \\
\hline $\begin{array}{l}\text { Not } \\
\text { Affiliated } \\
\text { with Ohio } \\
\text { State }\end{array}$ & 53 & 4.21 & 39 & 3.4 & 40 & 3.8 & 33 & 3.1 & 29 & 3.0 & 28 & 3.2 & 27 & 3.7 & $X$ & $X$ \\
\hline $\begin{array}{l}\text { A Spouse } \\
\text { of an Ohio } \\
\text { State } \\
\text { Employee }\end{array}$ & 40 & 75.4 & 29 & 74.4 & 28 & 70.0 & 28 & 84.9 & 23 & 79.3 & 21 & 75.0 & 19 & 70.4 & $X$ & $X$ \\
\hline
\end{tabular}

\section{Anxiety Findings}

In Week 1 of Series One, 17.5\% of participants felt that they were "not at all" nervous, anxious, or on edge, and this increased to $37.0 \%$ by Week 8; an increase of 20.5\% (Figure 1). The most frequently selected option for feeling nervous, anxious, or on edge was "several days a week" for both Series One (average of 52.0\%) and Series Two (49.0\%), and this remained consistent week by week. In terms of feeling anxious "nearly every day," 11.97\% participants agreed with this sentiment in Week 1 of Series One and 6.84\% felt this way in Week 1 of Series Two. These percentages decreased more in Series One than Series Two by the end of Week 8, as 4.91\% of participants reported feeling this way in Series 1 and 6.5\% in Series Two. 
Figure 1. Frequency of Feeling Nervous, Anxious, or On Edge by Week (Series One and Series Two)
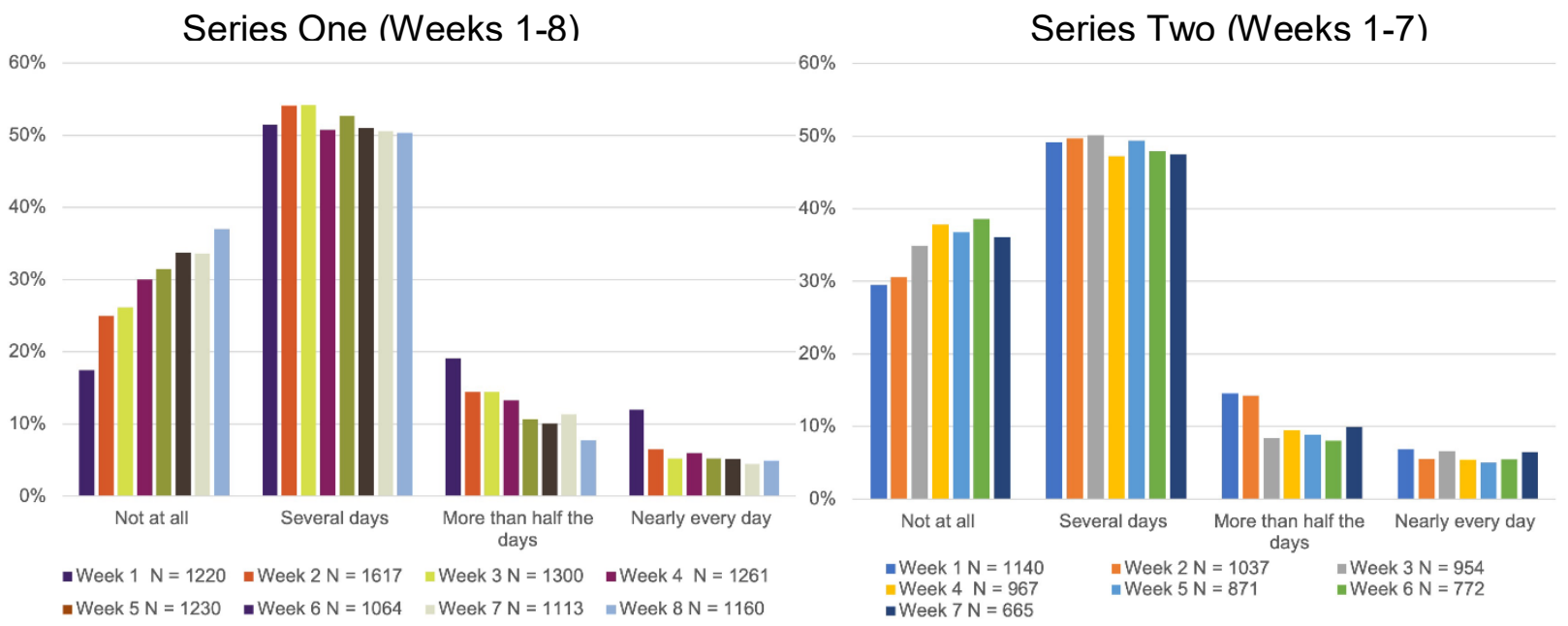

Figure 1. Sample size varied by week.

Not being able to stop or control worrying is an indicator of anxiety, and 40.5\% of Week 1, Series One participants were able to control their worry "all of the time," and this feeling increased to $60.0 \%$ after the eight-week series (Figure 2). In Series Two, Week 1, 51.3\% of participants could control their worry all of the time, and by week 7 this increased to $58.1 \%$. However, this left an average of $40.0 \%$ of participants who reported not being able to control the worry several days a week (Series One $=29.9 \%$; Series Two $=29.8 \%)$, more than half the days $(7.0 \% ; 7.6 \%)$, or nearly every day $(3.0 \% ; 4.5 \%)$ by the end of both series.

Figure 2. Frequency of Not Being Able to Stop or Control Worry (Series One and Series Two)

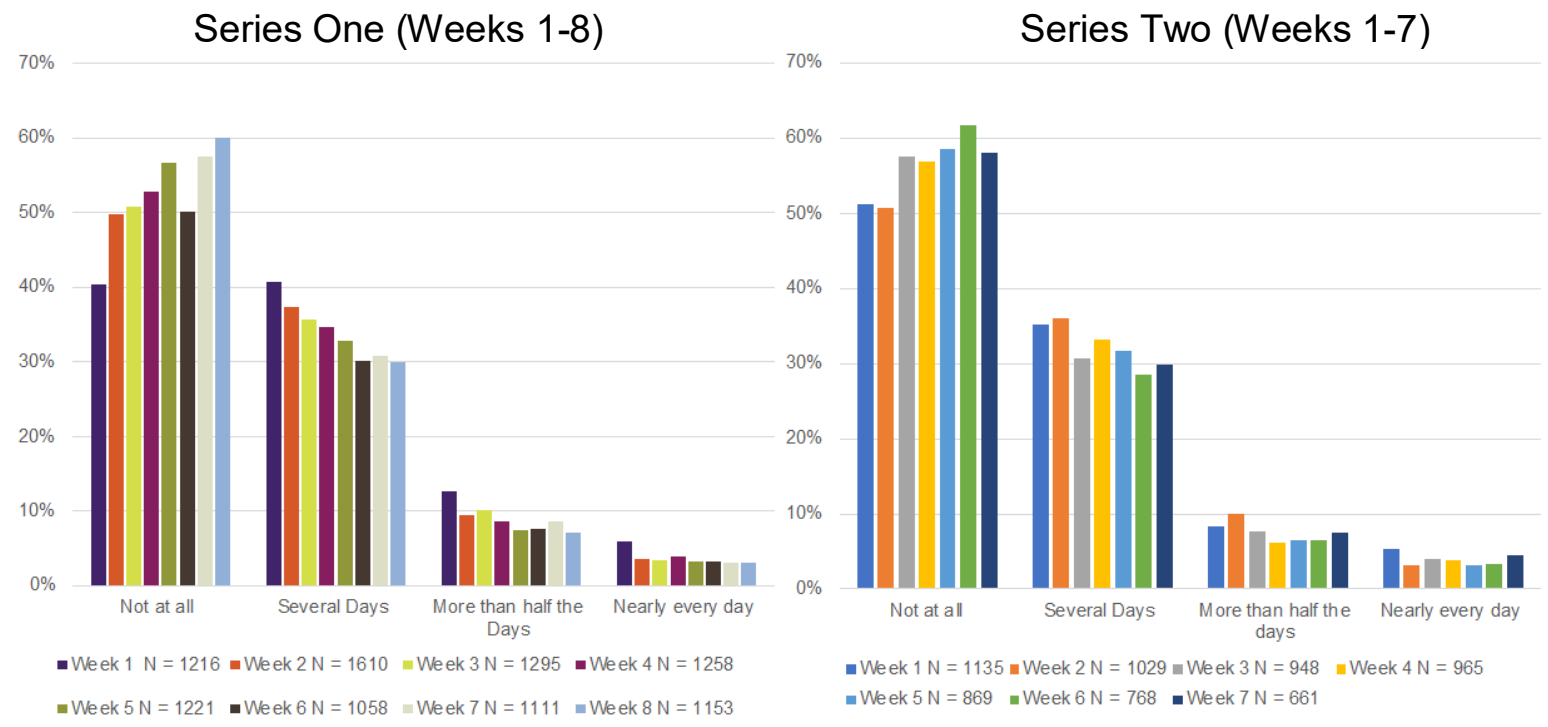

Figure 2. Sample size varied by week. 


\section{Depression Findings}

Similar to the trend for anxiety, the percentages for feeling depressed in Series One and Two decreased as the weeks continued (Figure 3 and 4). Most participants in Series One and Two reported no issues with having little interest or pleasure in doing things (an average of 60.0\% in Series One; 64.0\% in Series Two). In Series One, 40.0\% struggled with having little interest or pleasure in doing things (several days a week [33.0\%], more than half the days [7.3\%], or nearly every day [2.3\%]). This average decreased to 36\% in Series Two (either several days a week [26.0\%], more than half the days [6.0\%], or nearly every day [2.6\%]).

For the frequency of feeling down or depressed the most frequently selected option in both Series one (55\%) and two $(61 \%)$ was "not at all." The next most frequently selected frequency was several days a week $(33 \%$ series one; $32 \%$ series two). The percentage of "nearly every day feeling down or depressed" was recorded at an average of $2.43 \%$ in Series One. This percentage averaged $2.86 \%$ in of the second series, a $0.43 \%$ increase.

Figure 3. Frequency of Having Little Interest or Pleasure in Doing Things by Week (Series One and Series Two)
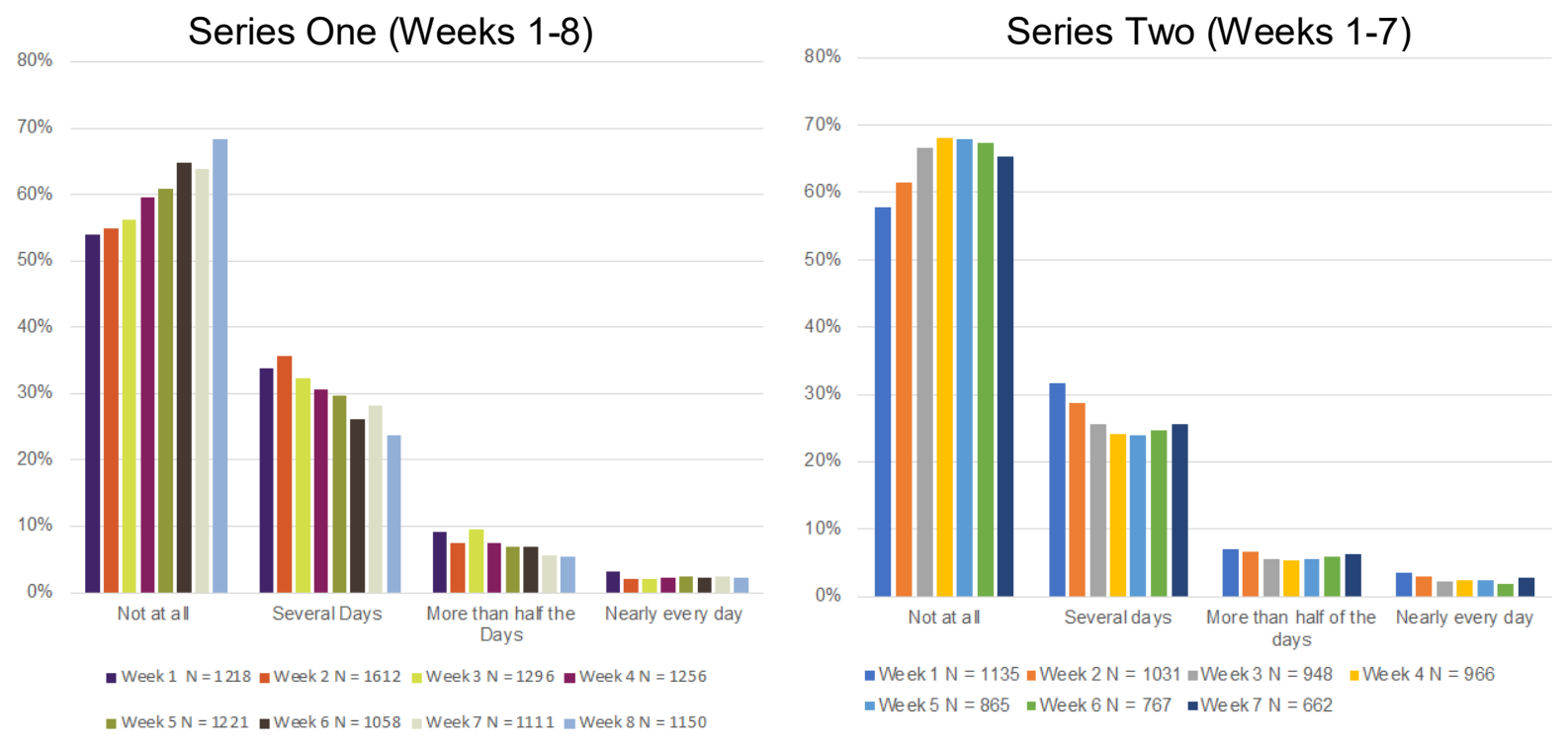

Figure 3. Sample size varied by week. 
Figure 4. Frequency of Feeling Down or Depressed by Week (Series One and Series Two)
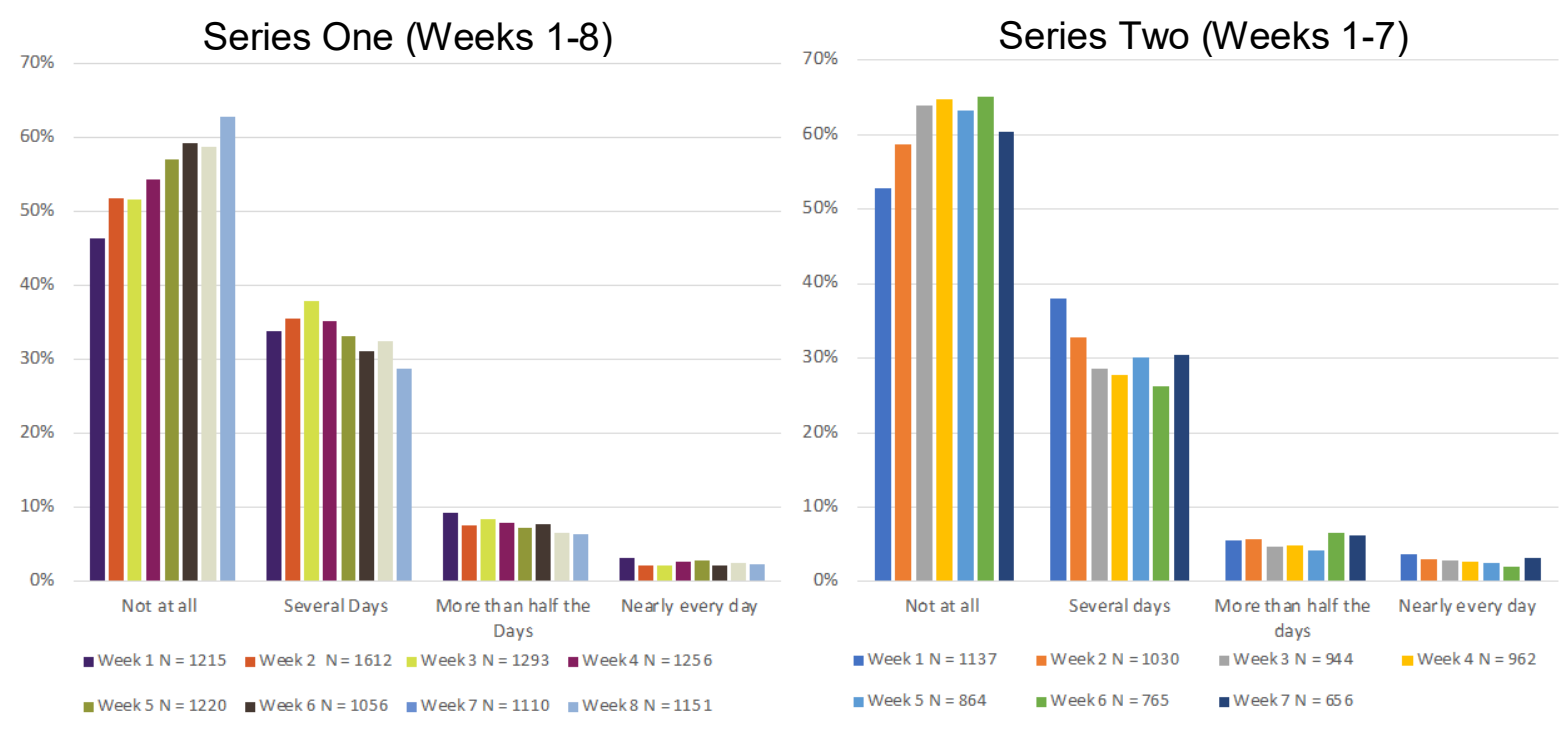

Figure 4. Sample size varied by week.

\section{Worry Thematic Analysis Findings}

During the final portion of the survey, participants were asked to answer the question "What are the 2 to 3 biggest concerns/worries that you have right now?" The questions produced a variety of responses at the individual level, but when reviewed as a whole, six major themes were identified: (1) Contracting COVID-19; (2) Finances (including job security); (3) Finding a Balance; (4) Health of Myself, Family, and Friends; (5) Mental Health; and (6) Politics/Civil Unrest. Response examples for each theme include "I'm concerned about getting sick" ; "[I am worried about] being able to pay bills on my income now that it's just my income" ; "[ $\mathrm{I}$ am $]$ overwhelmed with work and home life expectations" ; "[ I worry about my] lack of exercise, eating too much" ; " [I am concerned with] my partner's mental health and conflict at home" ; and "[I am worried about $]$ violence from the negativity promoted by politicians."

Contracting COVID-19 was a main issue due to its novelty and effect on everyday life. The uncertainty of COVID-19 caused a lot of worry in the participants. Participants were also very concerned about becoming sick, getting other family members sick, or their friends becoming sick. This worry was extremely prominent during Series One, and while these concerns remained in Series Two, it was not as frequent. As the months went on, participants still worried about COVID-19, but worries revolved around the government's handling of the pandemic and people not following appropriate preventative measures, such as wearing masks.

Finances were also a huge concern as the resulting lockdowns closed businesses across the globe. Participants worried about being laid off or furloughed, which would impact their families' financial security. These concerns become more prominent in Series Two as more participants reported spouses losing their jobs.

Participants frequently reported a lack of balance in their life in both Series One and Two. Life balance issues included managing childcare while working from home; the inability to focus while working from home; too many Zoom meetings; lacking an in person social life due to quarantine; and maintaining healthy behaviors while everything was shut down (i.e., healthy eating and appropriate physical activity). The lack of balance was commonly reported 
along with the mental health concerns as participants struggled to manage all the priorities in their lives. Competing interests between work, family, and friends caused significant feelings of anxiety and depression.

Lastly, prominent themes of displeasure with the federal government and concern for the racial divide began to appear after the tragic killing of George Floyd at the end of May 2020 and resulting protests in June 2020. These concerns from Series One continued into Series Two and increased in frequency in relation to the continued protests, lack of justice for Breonna Taylor, high stakes presidential election, and failure to pass another COVID relief bill.

\section{DICUSSION}

These results indicate a trend in anxiety and depressive symptom reduction over time. In both webinar series, the frequency of participants who reported "not at all" for experiencing the anxiety and depressive symptoms listed in PHQ-4 increased from the first week to the final week of the series, and those who reported several days, more than half the days, and nearly every day decreased. The reductions in symptomology persisted even though participant worry trends remained consistent (as seen in the worry theme analysis). These findings suggest that the evidencebased series content (e.g., cognitive-behavioral skills building and positive psychology) may have assisted in the participants' ability to emotionally regulate.

Cognitive-behavioral skills and positive psychology tips were taught in week 1 Series One and week 2 Series Two, respectively. Cognitive-behavioral skills are rooted in cognitive behavioral therapy (the gold standard evidence-based treatment for depression and anxiety treatment) which focuses on how thoughts impact feelings and behaviors (i.e., the thinking/feeling/behaving triangle; David, Cristea, \& Hoffman 2018; Fordham et al., 2021). Accordingly, participants were taught and then practiced how to identify their stressful events, how to recognize the negative thoughts that follow, and how to replace those negative thoughts with neutral or positive thoughts to ultimately change their behavior and emotional outcomes. Positive psychology uses the PERMA (positive emotions, engagement, relationships, meaning, and achievement) Model for building happiness into one's life (Seligman, 2019). Small simple actions like practicing gratitude, positive journaling, and calling friends were reviewed as helpful actions for meeting PERMA with webinar participants. Participants were also asked during a breakout activity to map out what PERMA meant to them and how they could accomplish it via small and attainable goals. Previous research has determined that both cognitive behavioral skills building and positive psychology can result in decreased anxiety and depression (Carr et al., 2020; Cunha, Pellanda, \& Reppold, 2019; Melnyk, Hoying, \& Tan, 2020; Sampson, Melnyk, \& Hoying, 2020).

While mental health improvements were made over the course of the webinars, the rates of experiencing symptoms of anxiety and depression for several days over the past two weeks remained around 25\% to 30\% by the end of both series (except frequency of feeling nervous, anxious, or on edge in series one which only dropped from $52 \%$ to 50\%), which is still high, but similar to the national averages reported during the COVID-19 pandemic by the Morbidity and Mortality Weekly Report (MMWR; Czeisler et al., 2020). However, the MMWR did note that reported anxiety and depression rates were 3 to 4 times higher than the rates reported in 2019, thus highlighting how a cooccurring mental health epidemic has been occurring within the COVID-19 pandemic. Digital tools, like the reviewed webinar, for health promotion are vital during this time as they provide mental health provisions effectively in a socially distanced manner (Rauschenberg et al., 2021). 


\section{Limitations}

While this study provided an excellent overview of webinar participant mental health status during the first six months of the COVID-19 pandemic, there were limitations. First, the amount of survey responses received varied by week and because the data was anonymous the study team was unable to confirm if the same participants tuned in weekly to watch the webinars and respond to the surveys. This likely introduced sampling and non-response bias. However, the anonymity of the survey provided a safe space in which participants could answer the questions honestly. Second, the study population was one of convenience and responses were mostly from faculty and staff members, therefore the results should not be generalized to other universities across the nation. Lastly, while there appeared to be a trend in reduction of anxiety and depressive symptoms over time, cause and effect cannot be inferred as other outside factors may have impacted the participants' responses.

\section{CONCLUSION}

Overall, participants in the Staying Calm and Well in the Midst of the COVID-19 Storm had a reduction in symptoms associated with anxiety and depression after completing the webinar series. However, roughly a fourth to a third of participants still reported having anxiety and depressive symptoms for several days after completing the series. As a result, the university wellness team is continuing to emphasize and provide evidence-based mental health programming and resources for its faculty, staff, and students. 


\section{REFERENCES}

Barbaresko, J., Rienks, J., \& Nöthlings, U. (2018). Lifestyle indices and cardiovascular disease risk: A metaanalysis. American Journal of Preventive Medicine, 55(4), 555-564. https://doi.org/10.1016/j.amepre.2018.04.046

Bin Zarah, A., Enriquez-Marulanda, J., \& Andrade, J. M. (2020). Relationship between dietary habits, food attitudes and food security status among adults living within the United States three months post-mandated quarantine: A cross-sectional study. Nutrients, 12(11), 3468. https://doi.org/10.3390/nu12113468

Carr, A., Cullen, K., Keeney, C., Cannig, C., Mooney, O., Chinseallaigh, E., \& O’Dowd, A. (2020). Effectiveness of positive psychology interventions: A systematic review and meta-analysis. The Journal of Positive Psychology, 121. https://doi.org/10.1080/17439760.2020.1818807

Centers for Disease Control and Prevention. (2020). Heart disease and mental health disorders. Retrieved from https://www.cdc.gov/heartdisease/mentalhealth.htm

Cunha, L. F., Pellanda, L. C., \& Reppold, C. T. (2019). Positive psychology and gratitude interventions: A randomized clinical trial. Frontiers in Psychology, 10, 584. https://doi.org/10.3389/fpsyg.2019.00584

Czeisler, M. É., Lane, R. I., Petrosky, E., Wiley, J. F., Christensen, A., Njai, R., .. Rajaratnam, S. (2020). Mental health, substance use, and suicidal ideation during the COVID-19 pandemic - United States, June 24-30, 2020. Morbidity and Mortality Weekly Report, 69(32), 1049-1057. https://doi.org/10.15585/mmwr.mm6932a1

David, D., Cristea, I., \& Hofmann, S. G. (2018). Why cognitive behavioral therapy is the current gold standard of psychotherapy. Frontiers in Psychiatry, 9, 4 https://doi.org/10.3389/fpsyt.2018.00004

Di Renzo, L., Gualtieri, P., Pivari, F., Soldati, L., Attinà, A., Cinelli, G., ... De Lorenzo, A. (2020). Eating habits and lifestyle changes during COVID-19 lockdown: An Italian survey. Journal of Translational Medicine, 18(1), 229. https://doi.org/10.1186/s12967-020-02399-5

Fordham, B., Sugavanam, T., Edwards, K., Stallard, P., Howard, R., Das Nair, R., ... Lamb, S. E. (2021). The evidence for cognitive behavioural therapy in any condition, population or context: A meta-review of systematic reviews and panoramic meta-analysis. Psychological Medicine, 51(1), 21-29. https://doi.org/10.1017/s0033291720005292

Institute of Medicine, \& National Research Council. 2015. Measuring the risks and causes of premature death: Summary of workshops. Washington, DC: The National Academies Press. https://doi.org/10.17226/21656.

Kent K., Goetzel R.Z., Roemer E.C., Prasad A., Freundlich N. (2016). Promoting healthy workplaces by building cultures of health and applying strategic communications. Journal of Occupational \& Environmental Medicine, 58(2),114-122. https://doi.org/10.1097/JOM.0000000000000629

Kirzinger, A., Kearney, A., Hamel, L., \& Brodie, M. (2020). KFF health tracking poll - early April 2020: The impact of Coronavirus on life in America. Kaiser Family Foundation. Retrieved from https://www.kff.org/coronavirus-covid-19/report/kff-health-tracking-poll-early-april-2020/

Kishore, S., Ripp, J., Shanafelt, T., Melnyk, B. M., Rogers, D., Brigham, T., .. Dzau, V. (26 October 2018). Making the case for the chief wellness officer in America's health systems: A call to action. Health Affairs Blog. Retrieved from https://www.healthaffairs.org/do/10.1377/hblog20181025.308059/full/ 
Kroenke, K., Spitzer, R. L., \& Williams, J. B. (2003). The Patient Health Questionnaire-2: Validity of a two-item depression screener. Medical Care, 41(11), 1284-1292. https://doi.org/10.1097/01.MLR.0000093487.78664.3C

Kroenke, K., Spitzer, R. L., Williams, J. B., Monahan, P. O., \& Löwe, B. (2007). Anxiety disorders in primary care: prevalence, impairment, comorbidity, and detection. Annals of Internal Medicine, 146(5), 317-325. https:/ / doi.org/10.7326/0003-4819-146-5-200703060-00004

Löwe, B., Wahl, I., Rose, M., Spitzer, C., Glaesmer, H., Wingenfeld, K., Schneider, A., \& Brähler, E. (2010). A 4item measure of depression and anxiety: Validation and standardization of the Patient Health Questionnaire4 (PHQ-4) in the general population. Journal of Affective Disorders, 122(1-2), 86-95. https://doi.org/10.1016/j.jad.2009.06.019

Marty, L., de Lauzon-Guillain, B., Labesse, M., \& Nicklaus, S. (2021). Food choice motives and the nutritional quality of diet during the COVID-19 lockdown in France. Appetite, 157, 105005. https://doi.org/10.1016/j.appet.2020.105005

Melnyk, B. M., Hoying, J., \& Tan, A. (2020). Effects of the MINDSTRONGC CBT-based program on depression, anxiety and healthy lifestyle behaviors in graduate health sciences students. Journal of American College Health, 1-9. Advance online publication. https://doi.org/10.1080/07448481.2020.1782922

Melnyk, B. M., \& Neale, S. (2019). Key strategies for optimizing personal health and well-being: A necessity for effective leadership. In B. M. Melnyk, \& T. Raderstorf (Eds.), Evidence-based leadership, innovation and entrepreneurship in nursing and healthcare: A practical guide to success (pp. 69-83). New York, New York: Springer Publishing Company, LLC. https://doi.org/10.1891/9780826196255.0004

Pfefferbaum, B., \& North, C. S. (2020). Mental Health and the Covid-19 Pandemic. The New England Journal of Medicine, 383(6), 510-512. https://doi.org/10.1056/NEJMp2008017

Ripp, J., \& Shanafelt, T. (2020). The health care chief wellness officer: What the role is and is not. Academic Medicine, 95(9), 1354-1358. https://doi.org/10.1097/ACM.0000000000003433

Rauschenberg, C., Schick, A., Hirjak, D., Seidler, A., Paetzold, I., Apfelbacher, C., Riedel-Heller, S. G., \& Reininghaus, U. (2021). Evidence synthesis of digital interventions to mitigate the negative impact of the COVID-19 Pandemic on public mental health: Rapid meta-review. Journal of Medical Internet Research, 23(3), e23365. https://doi.org/10.2196/23365

Sallis, J. F., Owen, N., \& Fisher, E. (2015). Ecological models of health behavior. In K. Glanz, B. K. Rimer, \& K. "V." Viswanath (Eds.), Health behavior: Theory, research, and practice (pp. 43-64). Hoboken, NJ: Jossey-Bass.

Sampson, M., Melnyk, B. M., \& Hoying, J. (2020). The MINDBODYSTRONG intervention for new nurse residents: 6-month effects on mental health outcomes, healthy lifestyle behaviors, and job satisfaction. Worldviews On Evidence-Based Nursing, 17(1), 16-23. http://doi.org/10.1111/wvn.12411

Seligman M. (2019). Positive Psychology: A Personal History. Annual Review of Clinical Psychology, 15, 1-23. https://doi.org/10.1146/annurev-clinpsy-050718-095653

Stockwell, S., Trott, M., Tully, M., Shin, J., Barnett, Y., Butler, L., ... Smith, L. (2021). Changes in physical activity and sedentary behaviours from before to during the COVID-19 pandemic lockdown: A systematic review. BMJ Open Sport \& Exercise Medicine, 7(e000960), 1-8. https://doi.org/10.1136/bmjsem-2020-000960

Uusitupa, M., Khan, T. A., Viguiliouk, E., Kahleova, H., Rivellese, A. A., Hermansen, K., Pfeiffer, A., Thanopoulou, A., Salas-Salvadó, J., Schwab, U., \& Sievenpiper, J. L. (2019). Prevention of type 2 diabetes by 
lifestyle changes: A systematic review and meta-analysis. Nutrients, 11(11), 2611. https://doi.org/10.3390/nu11112611

Address Author correspondence to:

Bernadette Mazurek Melnyk, PhD, APRN-CNP, FAANP, FNAP, FAAN

The Ohio State University

145 Newton Hall

1585 Neil Avenue Columbus, OH 43210

Melnyk.15@osu.edu 